\title{
Comparison of read-through effects of aminoglycosides and PTC124 on rescuing nonsense mutations of HERG gene associated with long QT syndrome
}

\author{
HAIYUN YU, XIAOYAN LIU, JIAN HUANG, YINHUI ZHANG, ROUMU HU and JIELIN PU \\ State Key Laboratory of Cardiovascular Disease, Physiology and Pathophysiology Laboratory, \\ Fuwai Hospital, National Center for Cardiovascular Diseases, Chinese Academy of Medical \\ Sciences and Peking Union Medical College, Beijing 100037, P.R. China
}

Received September 5, 2013; Accepted December 10, 2013

DOI: $10.3892 /$ ijmm.2013.1601

\begin{abstract}
Aminoglycosides promote the readthrough of premature stop codons introduced by nonsense mutations to produce full-length proteins in genetic disease models. The read-through effects of different aminoglycosides and PTC124 on HERG gene have yet to be adequately elucidated. The wild-type (WT) or mutant genes were transiently transfected in HEK293 cells. The read-through effect was examined by adding drugs into culture medium for $24 \mathrm{~h}$. Western blot analysis and patch clamping were performed to evaluate the expression and function of the genes. The mRNA levels were determined using qPCR. The results showed that G418 and PTC124 significantly increased the protein expression of R1014X mutant in a dose-dependent manner and produced a full-length protein. The maximal protein levels after G418, gentamicin or PTC124 treatment were $39.1 \pm 2.4,18.6 \pm 0.3$ or $10.3 \pm 1.0 \%$, respectively, of the WT level. Tobramycin did not exhibit a read-through effect. The mRNA levels, however, did not differ between WT and mutant gene. The tail current densities of R1014X channels at $40 \mathrm{mV}$ were $22.57 \pm 2.26 \mathrm{pA} / \mathrm{pF}$ for $\mathrm{G} 418,16.21 \pm 1.49 \mathrm{pA} / \mathrm{pF}$ for gentamicin and $9.62 \pm 0.73 \mathrm{pA} / \mathrm{pF}$ for PTC124. The leftward shift of the activation curve was corrected only by G418 and gentamicin. The read-through effects of W927X, R863X and E698X revealed that as the mutation site approached the $\mathrm{N}$-terminal, the rescue efficiency was decreased. The above results suggest that aminoglycosides and PTC124 induced different effects on rescue nonsense muta-
\end{abstract}

Correspondence to: Professor Jielin $\mathrm{Pu}$, State Key Laboratory of Cardiovascular Disease, Physiology and Pathophysiology Laboratory, Fuwai Hospital, National Center for Cardiovascular Diseases, Chinese Academy of Medical Sciences and Peking Union Medical College, 167 Bei-Li-Shi Road, Xi-Cheng, Beijing 100037, P.R. China

E-mail: jiayoucomeon@gmail.com

Key words: aminoglycosides, nonsense mutation, $I_{\mathrm{kr}}$, inherited arrhythmia, sudden cardiac death tions of the HERG gene. The mutation site was a significant factor in determining the pharmacological rescue efficiency.

\section{Introduction}

The human ether-a-go-go-related gene (HERG) gene encodes the $\alpha$ subunit of the rapid delayed rectifier potassium current channel $\left(I_{\mathrm{kr}}\right)$, which is important for cardiac repolarization. Mutations of the HERG gene that cause the dysfunction of $I_{\mathrm{kr}}$ may lead to type 2 long QT syndrome (LQTS). Approximately $10 \%$ of mutations are nonsense mutations which introduce the premature termination codons into HERG gene (PTC) $(1,2)$.

Aminoglycosides showed the read-through effect on nonsense mutations to produce full-lengh functional proteins in genetic disease models, such as cystic fibrosis and Duchenne muscular dystrophy (DMD) (3-6). It was previously demonstrated that gentamicin partially restored the HERG-like currents in HEK293 cells expressing R1014X mutant (7). However, whether other aminoglycosides had a similar effect, the most effective concentration as well as whether mutation sites affected the rescue effect remain to be determined.

PTC124 (3-[5-(2-fluorophenyl)-[1,2,4]oxadiazol-3-yl]benzoic acid; $\mathrm{C}_{15} \mathrm{H}_{9} \mathrm{FN}_{2} \mathrm{O}_{3}$ ) is a new option for numerous genetic diseases related to nonsense mutations (8-11). The chemical structure of PTC124 is different from aminoglycosides. It can act as a PTC suppressor to overcome the PTC and allow synthesis of a mature protein similar to gentamicin but without serious toxic side-effects (11).

The aim of this study was to evaluate the ability of three aminoglycosides (G418, gentamicin and tobramycin), which share a 4,6-disubstituted deoxystreptamine ring, and PTC124 to rescue nonsense mutations of HERG gene.

\section{Materials and methods}

Cell culture and transfection. Human embryonic kidney cells (HEK293) were grown in Dulbecco's modified Eagle's medium (DMEM) supplemented with $10 \%$ fetal bovine serum, $1 \%$ glutamine, $100 \mathrm{IU} / \mathrm{ml}$ penicillin, and $100 \mu \mathrm{g} / \mathrm{ml}$ streptomycin at $37^{\circ} \mathrm{C}$ in humidified $5 \% \mathrm{CO}_{2}, 95 \%$ air incubator. The wild-type (WT) HERG cDNA cloned into pcDNA3.1 vector was a kind 
gift from Dr Zhengfeng Zhou (Oregon Health and Science University, Portland, OR, USA). Four HERG nonsense mutations, R1014X, W927X, R863X and E698X were generated in the pcDNA3.1 WT HERG plasmid using polymerase chain reaction-based mutagenesis strategy and the sequence was then verified. Transfections were carried out with Effectene transfection reagent (Qiagen, Hilden, Germany) according to the manufacturer's instructions. HEK293 cells were transiently transfected with $0.4 \mu \mathrm{g}$ WT or equal amounts of mutant cDNA, respectively. Green fluorescent protein (GFP, $0.2 \mu \mathrm{g}$ ) gene was cotransfected as an indicator during patch clamp recording. Pharmacological rescue was examined by adding G418, gentamicin, tobramycin or PTC124 into DMEM for $24 \mathrm{~h}$.

Western blot analysis. After washing three times with PBS, cells were disrupted by adding lysis buffer containing PMSF and a phosphatase inhibitor mixture, followed by centrifugation at $12,000 \times \mathrm{g}$ for $10 \mathrm{~min}$ at $4^{\circ} \mathrm{C}$. The supernatant was obtained. Protein concentration was determined by using an Enhanced BCA protein assay kit (Beyotime, Nantong, China). Protein samples were then separated by $10 \%$ SDS-PAGE and transferred by semi-dry blotting to a nitrocellulose membrane and incubated with rabbit anti-N or anti-C terminal HERG antibodies (Alomone Laboratories Ltd., Jerusalem, Israel) overnight at $4^{\circ} \mathrm{C}$. The membranes were washed three times in TBST buffer, incubated with 1:2,500 dilutions of anti-rabbit IgG at room temperature for $1 \mathrm{~h}$, and again washed three times in TBST. Visualization was carried out using an enhanced chemiluminescence kit (Thermo Fisher Scientific Inc., Rockford, IL, USA). Band density on western blots was quantified by densitometry analysis of the scanned blots, using $\beta$-actin as the internal control.

$q P C R$. Cells were collected and total RNA was extracted by homogenization in TRIzol reagent according to the manufacturer's instructions. RNA was reverse transcribed. Quantitative polymerase chain reaction (qPCR) was prepared with PCR reagents and primers designed according to their recommendations, including HERG and GAPDH. The primer sequences for the genes were: HERG: (forward) 5'-GCTTGCTCAACT CCACCTC-3' and (reverse) 5'-TTTGGGGAATCTTGC TAATG-3'; GAPDH: (forward) 5'-CCCTTCATTGACCTC AACTACATG-3' and (reverse) 5'-CTTCTCCATGGTGG TGAAGAC-3'. qPCR was performed using $1 \mu \mathrm{l}$ of target cDNAs at $94^{\circ} \mathrm{C}$ for $2 \mathrm{~min}$, followed by 40 cycles at $94^{\circ} \mathrm{C}$ for $15 \mathrm{sec}$ and at $58^{\circ} \mathrm{C}$ for $30 \mathrm{sec}$ and $72^{\circ} \mathrm{C}$ for $15 \mathrm{sec}$ with the use of a SYBR-Green Master mix Kit. For all samples, GAPDH RNA was amplified using an internal control. Relative gene expression was calculated using the $2^{-\Delta \Delta C t}$ method.

Patch clamp recording. The whole-cell patch clamp method was applied in our experiment to record the HERG current. The bath solution contained the following (in $\mathrm{mM}$ ): $135 \mathrm{NaCl}$, $5 \mathrm{KCl}, 2 \mathrm{CaCl}_{2}, 1 \mathrm{MgCl}_{2} \cdot 6 \mathrm{H}_{2} \mathrm{O}, 10$ glucose, $10 \mathrm{HEPES}$. The internal pipette solution contained the following (in $\mathrm{mM}$ ): $140 \mathrm{KCl}, 0.5 \mathrm{MgCl}_{2} \cdot 6 \mathrm{H}_{2} \mathrm{O}, 5 \mathrm{HEPES}, 2$ EGTA, $4 \mathrm{~K}_{2}$ ATP. The HERG current was activated by a depolarizing pulse $(4 \mathrm{sec})$ to $50 \mathrm{mV}$ from the holding potential of $-80 \mathrm{mV}$. The tail current of the HERG channel was recorded following repolarization to $-50 \mathrm{mV}$. Voltage dependence of channel activation was obtained by fitting normalized curves with the Boltzmann function: $\mathrm{I} / \mathrm{Imax}=1 /(1+\exp [(\mathrm{V} 1 / 2-\mathrm{V}) / \mathrm{k}]): \mathrm{I}=$ measured tail current, Imax $=$ maximal tail current, $\mathrm{V}=$ applied membrane voltage, $\mathrm{V} 1 / 2=$ voltage at which half of the channels are activated, and $\mathrm{k}=$ slope factor. All the patch clamp experiments were performed at room temperature $\left(22 \pm 1^{\circ} \mathrm{C}\right)$.

Statistical analysis. Data are expressed as the means $\pm \mathrm{SE}$ and were analyzed by using SPSS v. 13.0 software. For statistical analysis, the Student's t-test was performed to compare between two data groups. For comparisons of data among groups, analysis of variance (ANOVA) was used. $\mathrm{P}<0.05$ was considered to indicate statistical significance.

\section{Results}

Nonsense mutations affected the protein expression but not transcription. By using antibody against N-terminus of the HERG protein, two protein bands were detected in the cells transfected with WT cDNA: the upper band at $155 \mathrm{kDa}$ and the lower band at $135 \mathrm{kDa}$ representing the complex glycosylated mature and core glycosylated immature form, respectively, of HERG proteins. There were also two bands in cells expressing R1014X and W927X mutants with molecular weights less than those of WT channels, representing their mature and immature form. The expression levels did not differ from those of WT. By contrast, the cells expressing R863X or E698X mutants, where mutation sites approached the N-terminal, only showed a single band with a molecular weight of $<100 \mathrm{kDa}$ and the protein expression levels were decreased significantly compared with those in cells expressing WT cDNA $(31.7 \pm 0.4 \%$ for R863X, $21.7 \pm 0.5 \%$ for E698X vs. $100 \%$ for WT, $\mathrm{n}=3$, both $\mathrm{P}<0.05$ ) (Fig. 1A).

The transcription of WT and four mutants (R1014X, W927X, R863X and E698X) were also examined. The mRNA levels were not different among cells transfected with either WT or mutants of HERG cDNA. The mRNA level was barely detectable in untransfected cells (Fig. 1B). The results above suggested that R863X and E698X mutations may have caused trafficking defect that inhibited the glycosylation process.

Nonsense mutations inhibited the HERG currents with different degrees. Ionic currents were recorded from HEK293 cells transiently transfected with WT cDNA and four mutants (Fig. 2A). The mean peak tail current densities of R1014X $(4.75 \pm 0.88 \mathrm{pA} /$ $\mathrm{pF}, \mathrm{n}=7)$ and $\mathrm{W} 927 \mathrm{X}(10.23 \pm 1.22 \mathrm{pA} / \mathrm{pF}, \mathrm{n}=7)$ were lower than those of WT channels $(52.83 \pm 4.01 \mathrm{pA} / \mathrm{pF}, \mathrm{n}=10$, both $\mathrm{P}<0.05)$, while there were no tail currents observed in cells transfected with R863X or E698X mutants (Fig. 2B). The voltage dependence of activation was negatively shifted in R1014X $\left(\mathrm{V}_{1 / 2}=0.20 \pm 1.13 \mathrm{mV}\right)$ and $\mathrm{W} 927 \mathrm{X}\left(\mathrm{V}_{1 / 2}=3.42 \pm 0.86 \mathrm{mV}\right)$ compared with that of the WT channel $\left(\mathrm{V}_{1 / 2}=10.67 \pm 0.68 \mathrm{mV}\right.$, both $\mathrm{P}<0.05)$, while the slope factors were similar in WT and mutant channels (Fig. 2C).

Aminoglycosides and PTC124 partially restored the production of full-length HERG protein from R1014X mutant. To examine the rescuing effect, three aminoglycosides and PTC124 were used. The 135- and 155-kDa bands were detected using antibodies against C-terminal of the HERG protein when the R1014X mutant was rescued, suggesting the full HERG protein was expressed. G418 promoted a significant effect of 
A
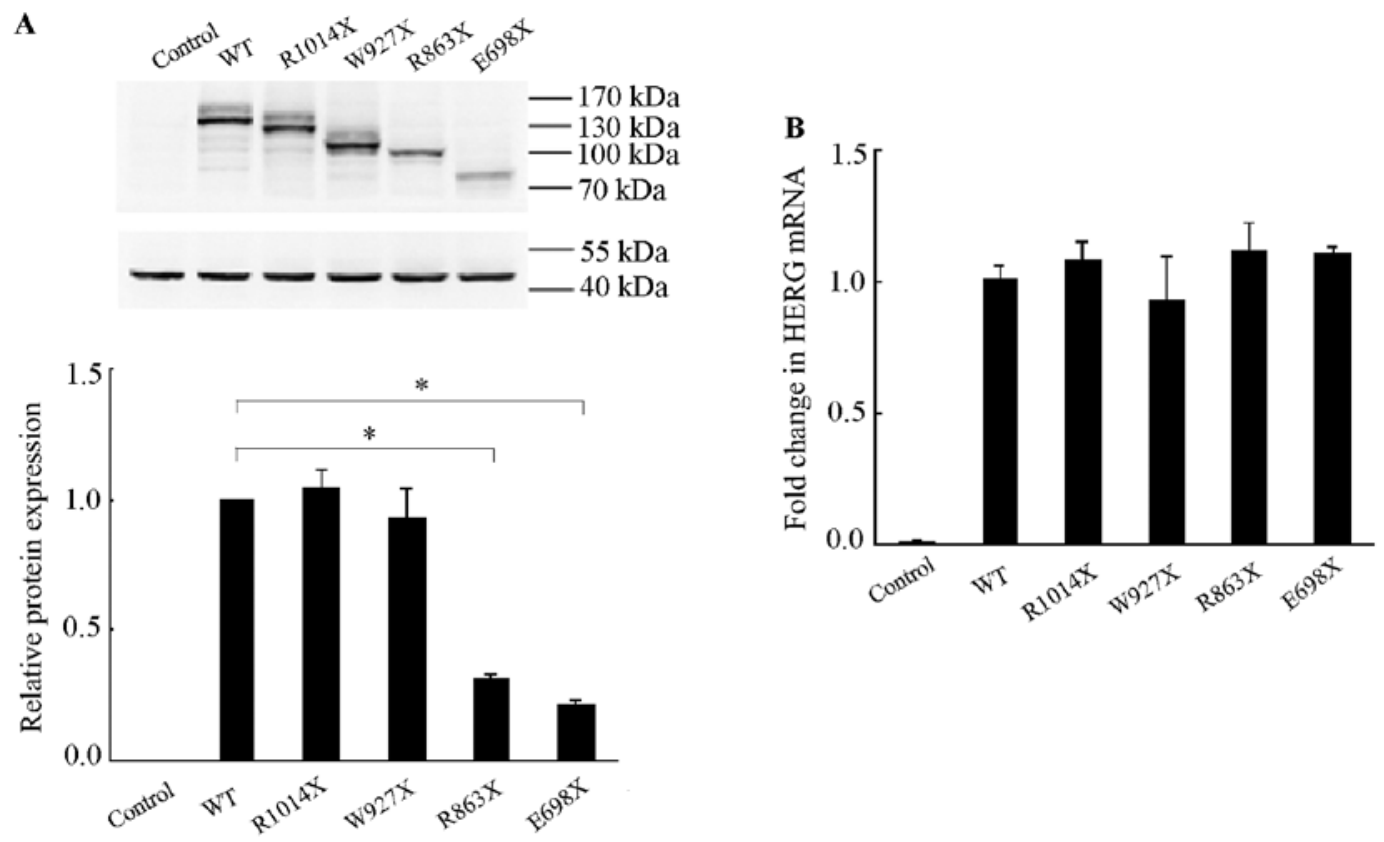

Figure 1. Expression of WT and mutant HERG proteins and mRNA in transfected HEK293 cells. (A) The different lengths of HERG protein were identified using antibody against $\mathrm{N}$-terminus. "P<0.05. (B) The mRNA level of four mutants was not markedly different from that of the WT gene.

A

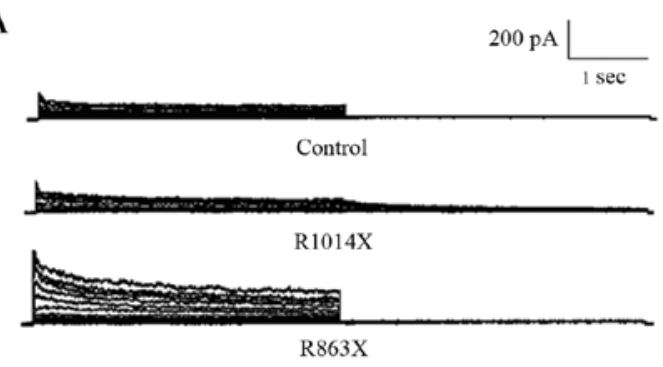

B

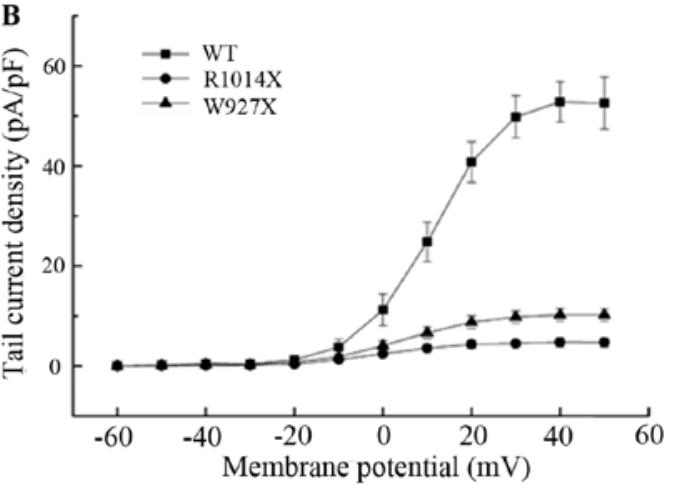

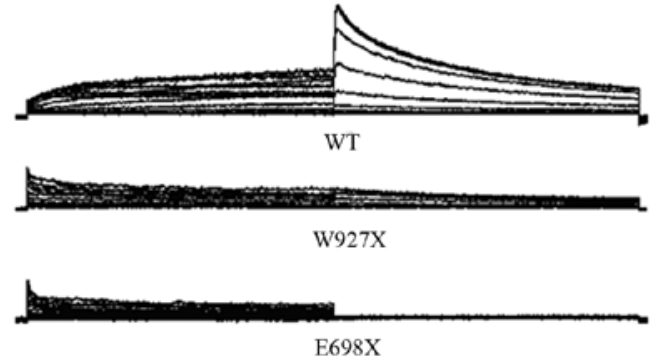

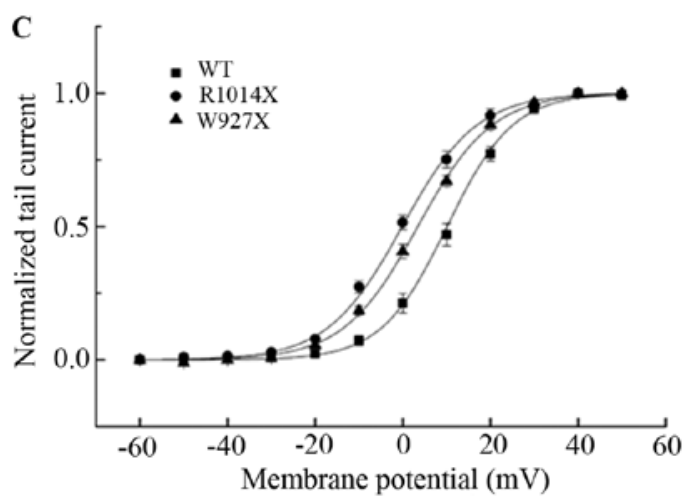

Figure 2. Ionic current recorded from WT and mutant HERG channels in transfected HEK293 cells. (A) Representative families of current traces recorded from cells transfected with WT and four mutant HERG cDNAs and the control (untransfected cells). (B) Current/voltage relationship of peak tail currents from cells transfected with WT, R1014X and W927X mutants. (C) The activation curves of peak tail current, normalized currents were plotted against the test potentials, and then fitted to the Boltzmann function. ${ }^{*} \mathrm{P}<0.05$.

readthrough in a dose-dependent manner $(0,14.3 \pm 1.1,25.4 \pm 1.1$ and $39.1 \pm 2.4 \%$ of the WT protein level, $\mathrm{n}=3, \mathrm{P}<0.05)$ under the concentrations of $0,50,100$ and $400 \mu \mathrm{g} / \mathrm{ml}$ (Fig. 3A and B). Gentamicin showed less maximal effect than G418, under the concentrations of $0,200,400$ and $800 \mu \mathrm{g} / \mathrm{ml}$, the WT protein levels were $0,11.8 \pm 0.7,18.6 \pm 0.3$ and $14.7 \pm 1.1 \%(n=3$, $\mathrm{P}<0.05$ ) (Fig. 3A and B). By contrast, tobramycin did not show any significant effect on rescuing R1014X mutant (Fig. 3A).
PTC124, a known compound for rescuing nonsense mutation, also showed read-through ability with lower efficiency $(0$, $2.9 \pm 0.6,7.6 \pm 0.5$ and $10.3 \pm 1.0 \%$ of the WT at concentrations of $0,10,50,200 \mu \mathrm{mol} / \mathrm{l}$ ) (Fig. 3A and B).

Then, we tested the effect of drugs on protein expression in cells transfected with WT-HERG gene by adding $\mathrm{G} 418(400 \mu \mathrm{g} / \mathrm{ml})$, gentamicin $(400 \mu \mathrm{g} / \mathrm{ml})$ and PTC124 ( $200 \mu \mathrm{mol} / \mathrm{l})$ into culture medium. The results showed there 

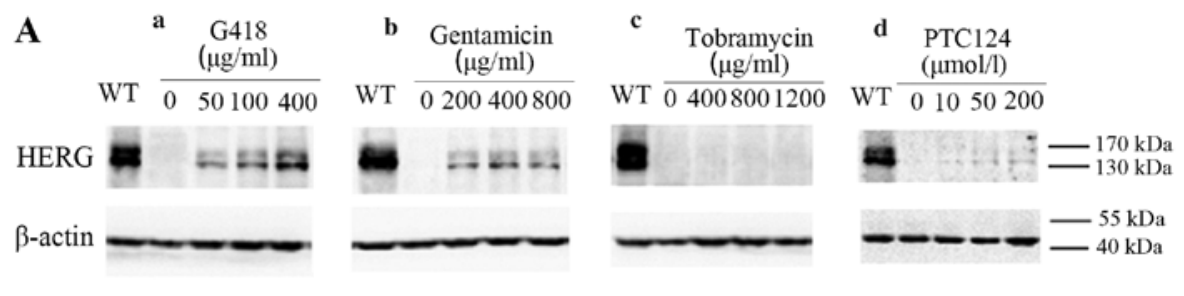

B

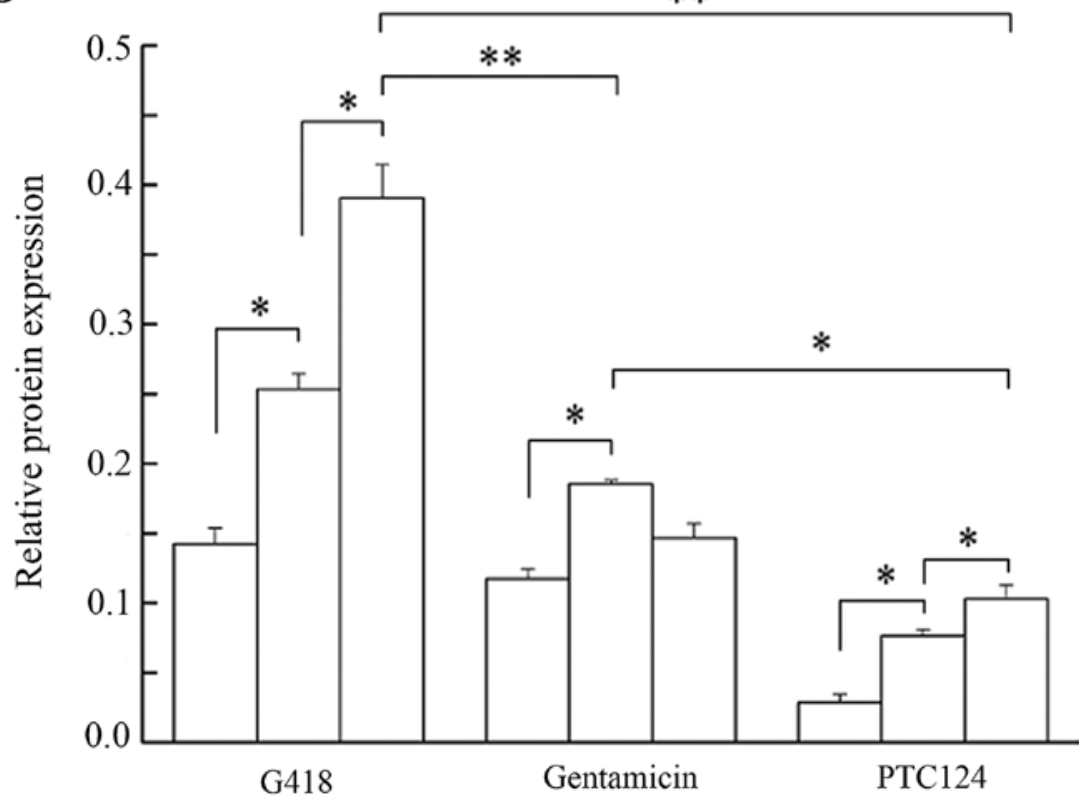

Figure 3. (A) Dose-dependent effects of readthrough of R1014X induced by (a) G418, (b) gentamicin, (c) tobramycin and (d) PTC124 were assessed using western blotting using $\beta$-actin as an internal control. (B) Cells were transfected with plasmids carrying WT or R1014X nonsense mutant, and then treated with different doses of G418, gentamicin, tobramycin or PTC124 to induce a read-through effect. ${ }^{*} \mathrm{P}<0.05,{ }^{, * *} \mathrm{P}<0.01$.

was no difference in protein expression levels in cells cultured with or without drugs (Fig. 4A and B), indicating that these drugs did not affect the WT-HERG expression.

Aminoglycosides and PTC124 restored current density and activation relation of R1014X mutant channel. The functional expression of R1014X mutant with and without drug treatment was tested by patch clamp recording (Fig. 5A). As shown in Fig. 5D, peak tail current density was increased in cells treated with $400 \mu \mathrm{g} / \mathrm{ml} \mathrm{G} 418(22.57 \pm 2.26 \mathrm{pA} / \mathrm{pF}, \mathrm{n}=6, \mathrm{P}<0.05)$ and gentamicin $400 \mu \mathrm{g} / \mathrm{ml}(16.21 \pm 1.49 \mathrm{pA} / \mathrm{pF}, \mathrm{n}=5, \mathrm{P}<0.05)$ compared to that of untreated cells $(4.75 \pm 0.88 \mathrm{pA} / \mathrm{pF}, \mathrm{n}=7)$, although this increase was lower than that of the WT channels $(52.83 \pm 4.01 \mathrm{pA} / \mathrm{pF}, \mathrm{n}=10)$. The leftward shift of the activation curve was corrected by the two drugs, i.e., $\mathrm{V}_{1 / 2}=12.05 \pm 1.93$ and $9.31 \pm 1.07$ for $\mathrm{G} 418$ and gentamicin, respectively, vs. and $0.20 \pm 1.13 \mathrm{mV}$ for the untreated cells, all $\mathrm{P}<0.05$ (Fig. 5C). PTC124 $(200 \mu \mathrm{mol} / \mathrm{l})$ treatment, however, only slightly increased the peak tail current (from $4.75 \pm 0.88 \mathrm{pA} / \mathrm{pF}$ to $9.62 \pm 0.73 \mathrm{pA} / \mathrm{pF}$, $\mathrm{n}=7, \mathrm{P}<0.05$; Fig. 5B and D) and did not correct the shift of the activation curve $\left(\mathrm{V}_{1 / 2}=0.20 \pm 1.13 \mathrm{mV}\right.$ vs. $\mathrm{V}_{1 / 2}=1.28 \pm 1.65 \mathrm{mV}$, $\mathrm{P}>0.05$; Fig. 5C). Tobramycin treatment, however, did not restore the current of R1014X mutant (Fig. 5A and B).

Aminoglycosides and PTC124 restored the protein expression of W927X but not R863X and E698X mutants. The read-through effects of G418, gentamicin and PTC124 on the
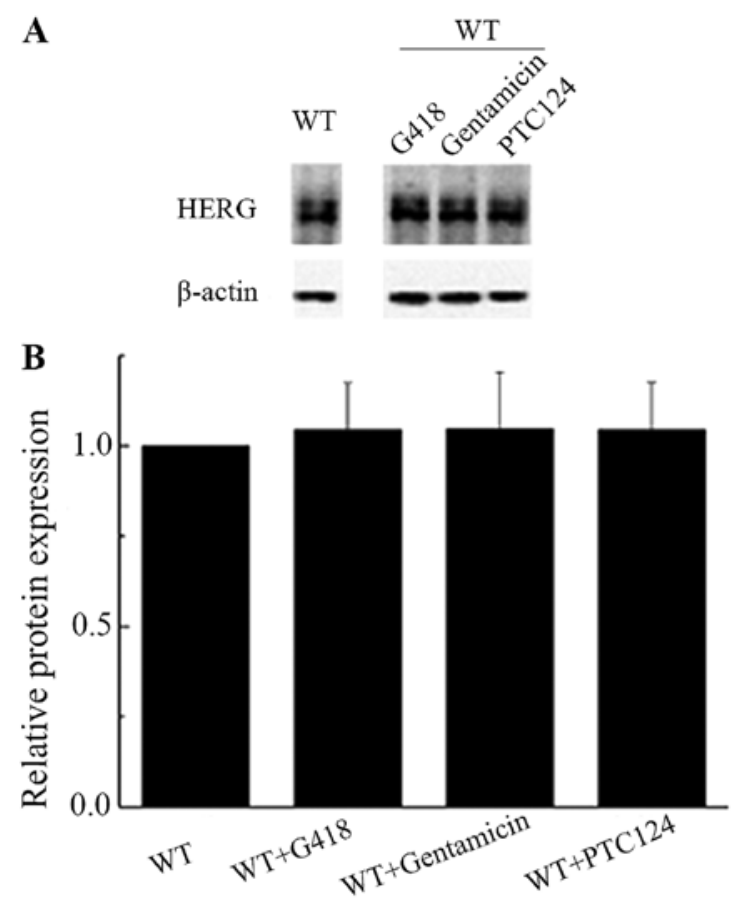

Figure 4. (A) Protein expression in cells transfected with WT-HERG gene by adding G418 $(400 \mu \mathrm{g} / \mathrm{ml})$, gentamicin $(400 \mu \mathrm{g} / \mathrm{ml})$ and PTC124 $(200 \mu \mathrm{mol} / \mathrm{l})$ into DMEM. Anti-N terminal antibody was used. $\beta$-actin was used as an internal control. When analyzing the HERG protein, anti-N terminal antibody was used. (B) There were no statistically significant differences observed among the groups when analyzing the HERG protein level. 
A

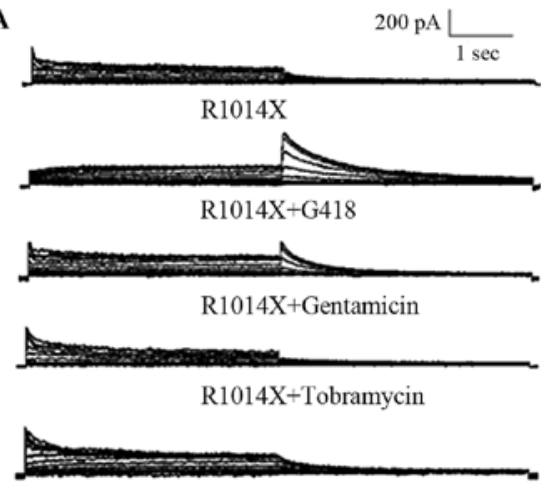

$\mathrm{R} 1014 \mathrm{X}+\mathrm{PTC} 124$

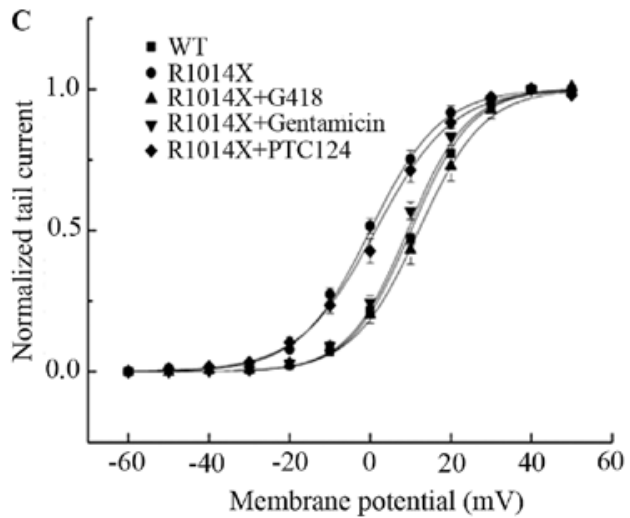

B

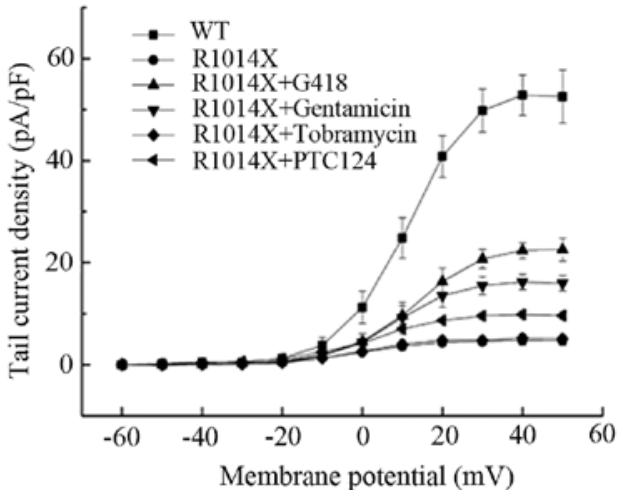

D

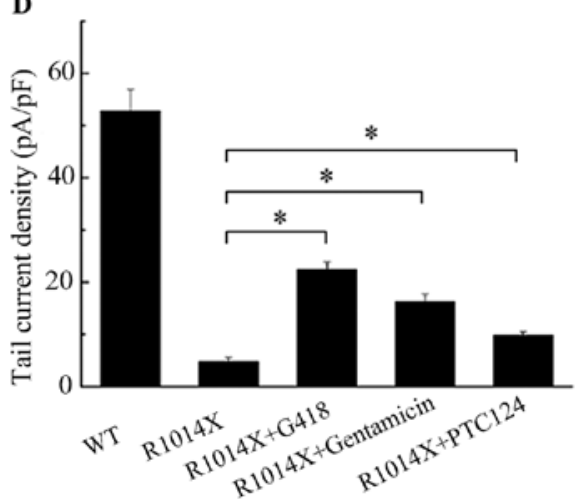

Figure 5. The pharmacological rescue of the functional expression of R1014X mutant. (A) Transfected cells were treated with G418, gentamicin, tobramycin and PTC124, respectively, examples of current recording obtained from each drug. (B) Current/voltage relationship of peak tail currents before and after pharmacological rescue. The activation curves of peak tail current with and without drug treatment. (C) Note that the leftward shift of the activation curve was corrected by G418 and gentamicn but not PTC124. "P<0.05. (D) Mean peak tail current densities of WT and R1014X with and without drugs.

A

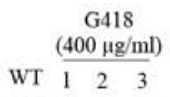

HERG

$\beta$-actin $-\infty-$

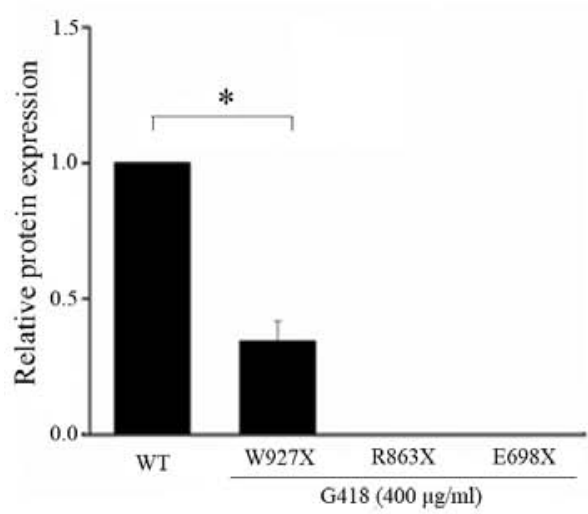

B
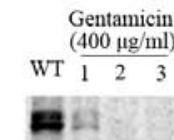

23
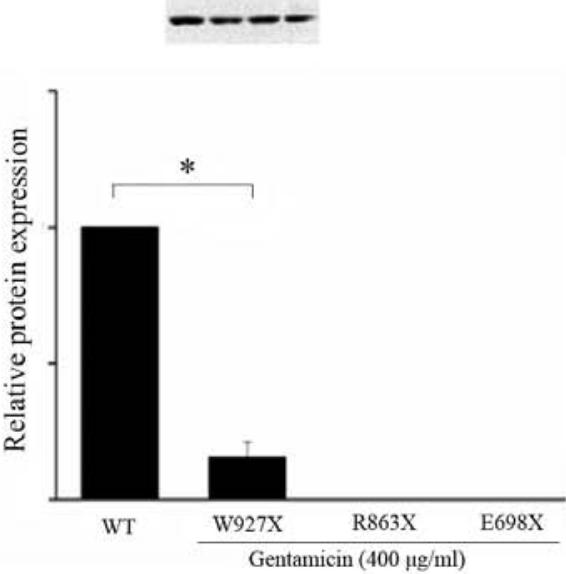

C
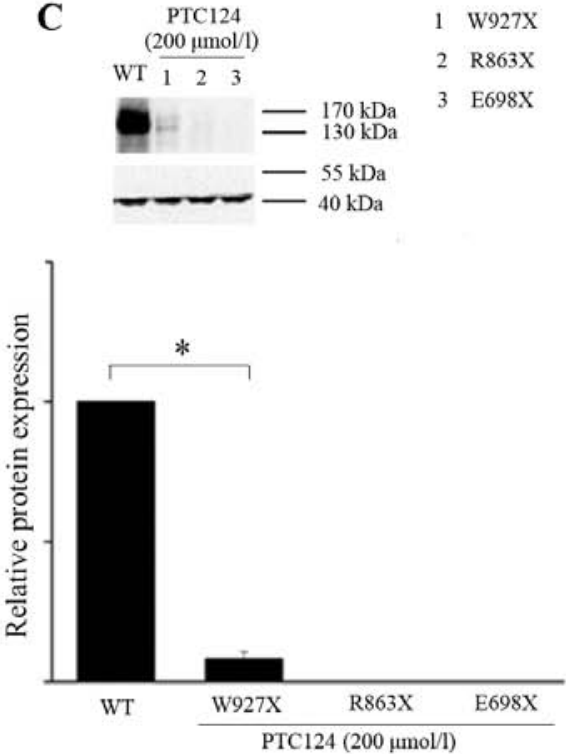

Figure 6. Mutation sites concerning read-through effects induced by (A) G418,(B) gentamicin and (C) PTC124. As the mutation site approached the N-terminal (R863X and E698X), the rescue effects of the three drugs were decreased. ${ }^{*} \mathrm{P}<0.05$.

expression of W927X, R863X and E698X mutants were tested. As shown in Fig. 6, all three compounds partially induced the expression of full-length protein in cells transfected with W927X mutant. The read-through efficiency of G418 was higher than that of gentamicin and PTC124 $(34.4 \pm 4.3 \%$ for G418 compared to $15.6 \pm 3.2$ and $8.3 \pm 1.3 \%$ for gentamicin and PTC124, respectively, $n=3$, both $\mathrm{P}<0.05$, Fig. 6). For R863X and E698X mutants, the read-through effect was not observed 
when the cells were treated by G418, gentamicin or PTC124, suggesting that as the mutation site approached the $\mathrm{N}$-terminal, the pharmacological rescue efficiency was decreased.

\section{Discussion}

In this study, we investigated the pharmacologic rescue effect on nonsense mutations of the HERG gene. The results showed that G418, gentamicin and PTC124 partially restored the expression of full-length protein in cells transfected with R1014X or W927X mutant. The leftward shift of the R1014X channel activation curve was also corrected subsequent to G418 and gentamicin treatment. Additionally, the mutation site was a factor affecting the rescuing efficiency.

LQTS is an inherited cardiac arrhythmia syndrome related to syncope and sudden death (12). It has been found that mutations in 13 encoding ion channels or structural protein genes are associated with LQTs. Mutations of the HERG gene lead to type 2 LQTs $(13,14)$.

In this study, we first observed the protein and mRNA levels of the WT-HERG gene and four nonsense mutants that produce C-terminus truncations. Results of the western blot analysis revealed that the untransfected HEK293 cells did not react with the antibody against the N-terminus of the HERG protein. Following transfection of cells with WT or mutant HERG cDNAs, different lengths of HERG proteins were detected according to mutation sites. As the mutation site approached the N-terminal, the protein became shorter. The protein level was decreased in cells transfected with R863X or E698X. Nonsense-mediated mRNA decay (NMD) is an effective mRNA surveillance mechanism which can detect and degrade mRNAs with premature termination condons (PTCs) and protects cells from the potentially deleterious effects of truncated proteins. However, the results of qPCR suggested an unchanged mRNA level, suggesting the mutants were not subjected to nonsense-mediated decay (NMD) mechanism in our expression system. A possible reason for this observation was that the cDNA did not contain the full configuration of exons and introns (15).

Residues $750-870$ of the HERG protein comprised the cyclic nucleotide binding domain (cNBD) which was responsible for protein trafficking (16). Disruption of this region may result in defect of protein trafficking. Residues 860-899 of the HERG protein were also reported to be essential for endoplasmic reticulum exit and for the stability of the channel protein (17). Nonsense mutation in this region may result in defective trafficking as well as rapid degradation of mutant channels in endoplasmic reticulum. Thus R863X and E698X only produced single bands and the protein level was significantly decreased compared to that of WT channels.

Aminoglycosides are classical antibiotics that bind to the A site of bacterial ribosomal 16S RNA to reduce translation fidelity, thus similar aminoacyl-tRNA enters the A site, inducing synthesis of error protein or preventing the release of protein and ultimately causing bacterial death (18). The crystal structure analysis revealed that aminoglycosides combined with A1408 and G1491 16S RNA by forming hydrogen bonds to cause conformational changes, reducing its ability to identify aminoacyl-tRNA. The structure of the eukaryotic ribosome 18S RNA was a little different from the prokaryotes
16S RNA, with A1408 $\rightarrow$ G and G1491 $\rightarrow$ A substitution. Previous studies have demonstrated that aminoglycosides also bind to the 18S rRNA subunit, reducing discrimination of nearcognate tRNAs, although this interaction was less stable than that in bacteria (19-21). Due to the lack of upstream and downstream normal stop codon sequence and polyA tail structure, the premature stop codon (PTC) could be suppressed more readily by aminoglycosides (22).

PTC suppression to restore the function of mutant proteins in mammalian cells was first reported by Howard et al (6). Their results showed that G418 suppressed PTCs to restore the function of mutant protein in transiently expressed cDNAs which contained mutations in the cystic fibrosis transmembrane conductance regulator (CFTR) gene. The potency of aminoglycosides for inducing the production of full-length proteins from other genes carrying a PTC, such as P53, APC and DMD genes has also been demonstrated $(4,23,24)$. In a previous study we have demonstrated that gentamicin suppressed PTC in R1014X and W927X mutants of the HERG gene to produce full-length channel protein (7).

Aminoglycosides were mainly divided into two categories: one with a 4,6-disubstituted deoxystreptamine ring and the other with a 4,5-disubstituted deoxystreptamine ring. In this experiment, we mainly explored the read-through effect of G418, gentamicin and tobramycin, which belong to the 4,6-disubstituted type. Our results suggest that G418 and gentamincin effectively rescued the R1014X mutant to produce full-length and functional protein. However, tobramycin was unable to show a significant read-through effect, consistent with the results of Manuvakhova et al (25). The authors of that study found that four compounds in the 4,6-disubstituted class (kanamycin A, kanamycin B, tobramycin, and amikacin) did not rescue the nonsense mutants constructed for that experiment. As the four compounds shared a common ring 3 that was not found on G418 and gentamicin, they suggested that certain characteristics of the ring 3 may have prevented the induction of the readthrough effect.

The severe side-effects such as nephrotoxicity and ototoxicity of aminoglycosides may limit their clinical application. Recently it has been reported that PTC124, which has low toxicity, may also act as a nonsense codon suppressor (10) in the studies using cell lines and mouse models with Duchenne muscular dystrophy and cystic fibrosis $(11,26)$. The effect of PTC124 on clinical trial in CF patients with nonsense mutations has also been demonstrated (27). In this study it has been demonstrated that PTC124 promoted the production of full-length protein in cells expressing R1014X mutant with lower efficiency, in contrast to previous studies which showed that PTC124 was more effective than gentamicin. By contrast, results of in vitro studies demonstrated readthrough induced by aminoglycosides but not PTC124 (28-30), suggesting specificity of PTC124 for particular genes.

In this experiment, we also tested the rescuing ability of G418, gentamicin and PTC124 on W927X, R863X and E698X mutants. As the mutation site approached the $\mathrm{N}$-terminal, the rescue ability was decreased. These results were different from studies in other genetic diseases $(23,31)$. The exact mechanism accounting for the phenomenon was unclear. Earlier studies indicated that tryptophan or glutamine may be inserted into the premature stop codon site $(32,33)$. We proposed that the 
amino acid that was inserted into the premature termination codon site induced by the read-through mechanism may be different from the original one in WT protein. Thus, it may result in the dysfunction of cNBD domain or residues 860-899, leading to trafficking defect.

Other aminoglycosides, as well as aminoglycoside derivatives with lower toxicity such as NB54 and NB84 have not been studied in our experiments $(25,34)$. Additionally, our study was limited to channels expressed in the homologous cell system. Additionaly studies are required to obtain an improved expression system or animal models to confirm our findings.

In conclusion, aminoglycosides and PTC124 induced different read-through effects on the nonsense mutations of HERG gene. G418 and gentamicin had a higher ability to rescue the nonsense mutants. The mutation site was a significant factor determining the rescue efficiency. As the mutation site approached the N-terminal, the rescue efficiency was decreased. These findings suggest complexity in the pharmacological treatment of diseases caused by nonsense mutations in the clinical setting.

\section{Acknowledgements}

This study was funded by the National Natural Science Foundation of China (project no., 81070150, to J. Pu).

\section{References}

1. Splawski I, Shen J, Timothy KW, et al: Spectrum of mutations in long-QT syndrome genes. KVLQT1, HERG, SCN5A, KCNE1, and KCNE2. Circulation 102: 1178-1185, 2000

2. Anderson CL, Delisle BP, Anson BD, et al: Most LQT2 mutations reduce Kv11.1 (hERG) current by a class 2 (trafficking-deficient) mechanism. Circulation 113: 365-373, 2006.

3. Lee HL and Dougherty JP: Pharmaceutical therapies to recode nonsense mutations in inherited diseases. Pharmacol Ther 136 227-266, 2012

4. Zilberberg A, Lahav L and Rosin-Arbesfeld R: Restoration of APC gene function in colorectal cancer cells by aminoglycosideand macrolide-induced read-through of premature termination codons. Gut 59: 496-507, 2010

5. Malik V, Rodino-Klapac LR, Viollet L, et al: Gentamicininduced readthrough of stop codons in Duchenne muscular dystrophy. Ann Neurol 67: 771-780, 2010.

6. Howard M, Frizzell RA and Bedwell DM: Aminoglycoside antibiotics restore CFTR function by overcoming premature stop mutations. Nat Med 2: 467-469, 1996

7. Yao Y, Teng S, Li N, Zhang Y, Boyden PA and Pu J: Aminoglycoside antibiotics restore functional expression of truncated HERG channels produced by nonsense mutations. Heart Rhythm 6: 553-560, 2009.

8. Wilschanski M, Miller LL, Shoseyov D, et al: Chronic ataluren (PTC124) treatment of nonsense mutation cystic fibrosis. Eur Respir J 38: 59-69, 2011.

9. Goldmann T, Overlack N, Wolfrum U and Nagel-Wolfrum K: PTC124-mediated translational readthrough of a nonsense mutation causing Usher syndrome type 1C. Hum Gene Ther 22: 537-547, 2011

10. Tan L, Narayan SB, Chen J, Meyers GD and Bennett MJ: PTC124 improves readthrough and increases enzymatic activity of the CPT1A R160X nonsense mutation. J Inherit Metab Dis 34: 443-447, 2011.

11. Welch EM, Barton ER, Zhuo J, et al: PTC124 targets genetic disorders caused by nonsense mutations. Nature 447: 87-91, 2007.

12. Crotti L, Celano G, Dagradi F and Schwartz PJ: Congenital long QT syndrome. Orphanet J Rare Dis 3: 18, 2008.

13. Curran ME, Splawski I, Timothy KW, Vincent GM, Green ED and Keating MT: A molecular basis for cardiac arrhythmia: HERG mutations cause long QT syndrome. Cell 80: 795-803, 1995.
14. Alders M and Mannens M: Romano-Ward Syndrome. Pagon RA, Adam MP, Bird TD, et al (eds.). GeneReviews ${ }^{\mathrm{TM}}$ (internet). University of Washington, Seattle, WA, 2003. http://www.ncbi. nlm.nih.gov/books/NBK1129/. Last update: May 31, 2012.

15. Schweingruber C, Rufener SC, Zünd D, Yamashita A and Mühlemann O: Nonsense-mediated mRNA decay - mechanisms of substrate mRNA recognition and degradation in mammalian cells. Biochim Biophys Acta 1829: 612-623, 2013.

16. Akhavan A, Atanasiu R, Noguchi T, Han W, Holder N and Shrier A: Identification of the cyclic-nucleotide-binding domain as a conserved determinant of ion-channel cell-surface localization. J Cell Sci 118: 2803-2812, 2005.

17. Akhavan A, Atanasiu R and Shrier A: Identification of a $\mathrm{COOH}-$ terminal segment involved in maturation and stability of human ether-a-go-go-related gene potassium channels. J Biol Chem 278: 40105-40112, 2003.

18. Moazed D and Noller HF: Interaction of antibiotics with functional sites in 16S ribosomal RNA. Nature 327: 389-394, 1987.

19. Kondo J, Urzhumtsev A and Westhof E: Two conformational states in the crystal structure of the Homo sapiens cytoplasmic ribosomal decoding A site. Nucleic Acids Res 34: 676-685, 2006.

20. Kondo J, Francois B, Urzhumtsev A and Westhof E: Crystal structure of the Homo sapiens cytoplasmic ribosomal decoding site complexed with apramycin. Angew Chem Int Ed Engl 45: 3310-3314, 2006.

21. Hermann T, Tereshko V, Skripkin E and Patel DJ: Apramycin recognition by the human ribosomal decoding site. Blood Cells Mol Dis 38: 193-198, 2007.

22. Amrani N, Ganesan R, Kervestin S, Mangus DA, Ghosh S and Jacobson A: A faux 3'-UTR promotes aberrant termination and triggers nonsense-mediated mRNA decay. Nature 432: 112-118, 2004.

23. Floquet C, Deforges J, Rousset JP and Bidou L: Rescue of non-sense mutated p53 tumor suppressor gene by aminoglycosides. Nucleic Acids Res 39: 3350-3362, 2011.

24. De Luca A, Nico B, Rolland JF, et al: Gentamicin treatment in exercised mdx mice: identification of dystrophin-sensitive pathways and evaluation of efficacy in work-loaded dystrophic muscle. Neurobiol Dis 32: 243-253, 2008.

25. Manuvakhova M, Keeling K and Bedwell DM: Aminoglycoside antibiotics mediate context-dependent suppression of termination codons in a mammalian translation system. RNA 6: 1044-1055, 2000.

26. Du M, Liu X, Welch EM, Hirawat S, Peltz SW and Bedwell DM: PTC124 is an orally bioavailable compound that promotes suppression of the human CFTR-G542X nonsense allele in a CF mouse model. Proc Natl Acad Sci USA 105: 2064-2069, 2008.

27. Kerem E, Hirawat S, Armoni S, et al: Effectiveness of PTC124 treatment of cystic fibrosis caused by nonsense mutations: a prospective phase II trial. Lancet 372: 719-727, 2008.

28. Ho G, Reichardt J and Christodoulou J: In vitro read-through of phenylalanine hydroxylase (PAH) nonsense mutations using aminoglycosides: a potential therapy for phenylketonuria. J Inherit Metab Dis: Mar 27, 2013 (Epub ahead of print).

29. Brumm H, Mühlhaus J, Bolze F, et al: Rescue of melanocortin 4 receptor (MC4R) nonsense mutations by aminoglycosidemediated read-through. Obesity (Silver Spring) 20: 1074-1081, 2012.

30. Dranchak PK, Di Pietro E and Snowden A, et al: Nonsense suppressor therapies rescue peroxisome lipid metabolism and assembly in cells from patients with specific PEX gene mutations. J Cell Biochem 112: 1250-1258, 2011.

31. Bartolomeo R, Polishchuk EV, Volpi N, Polishchuk RS and Auricchio A: Pharmacological read-through of nonsense ARSB mutations as a potential therapeutic approach for mucopolysaccharidosis VI. J Inherit Metab Dis 36: 363-371, 2013.

32. Engelberg-Kulka H: UGA suppression by normal tRNA Trp in Escherichia coli: codon context effects. Nucleic Acids Res 9: 983-991, 1981.

33. Nilsson $M$ and Ryden-Aulin M: Glutamine is incorporated at the nonsense codons UAG and UAA in a suppressor-free Escherichia coli strain. Biochim Biophys Acta 1627: 1-6, 2003.

34. Brendel C, Belakhov V, Werner H, et al: Readthrough of nonsense mutations in Rett syndrome: evaluation of novel aminoglycosides and generation of a new mouse model. J Mol Med (Berl) 89: 389-398, 2011. 